\title{
Three-dimensional Finite Element simulation of acoustic propagation in spiral bubble
}

net of humpback whale

Xin Qing, ${ }^{1, \text { a) }}$ Paul R. White, ${ }^{2}$ Timothy G. Leighton, ${ }^{2}$ Songzuo Liu, ${ }^{1, b}$ Gang Qiao, ${ }^{1, a}$ and Yu Zhang ${ }^{3}$

${ }^{1}$ Acoustic Science and Technology Laboratory, College of Underwater Acoustic Engineering, Harbin Engineering University, Harbin, 150001, China.

${ }^{2}$ Institute of Sound and Vibration Research, University of Southampton, Highfield, Southampton SO17 1BJ, United Kingdom

${ }^{3}$ Key Laboratory of Underwater Acoustic Communication and Marine Information Technology of the Ministry of Education, Xiamen University, College of Ocean and Earth Sciences, Xiamen, 361005, China

(Dated: 11 July 2019) 
In 2004, Leighton hypothesized that the acoustic calls emitted by humpback whales when feeding using bubble nets, may enhance the effectiveness of the net in confining prey (such as herring) by forming a 'wall of sound' with a quiet zone within. Modelling of the acoustics of this phenomenon was previously restricted to 2D; this paper conducts a 3D model of the propagation of signals resembling those emitted by humpback whales when bubble netting, projected into a upward spiral bubble net which data to data suggests is the accurate form for the bubble net in 3D space. In this study, the feeding calls were analyzed in the time-frequency domain to extract acoustic information sufficient to allow modeling of the resulting spatial distribution of acoustic pressure and particle velocity, and how they vary over the duration of the call. Sound propagation in the bubble net was described by using a linear steady-state formulation for an effective medium of bubbly water. Using the predicted attenuation, phase velocity and density in bubbly water, a 3D finite element model was constructed to numerically simulate the upward-spiral bubble net which consists of a mixture of bubbles that exhibit a range of radii. The acoustic pressure field and particle motion field were both calculated within the bubble net. The simulation results show that the energy of the whale feeding call could be effectively focused in the bubble net, generating intensive sound pressure and particle motion fields in the bubbly arm of the net, but with some 'quiet' regions closer to the center of the net, as Leighton hypothesized. Furthermore, when the hearing ability of herring is taken into consideration, the results suggest that this acoustic focusing effect could be a plausible factor in trapping them in the bubble net. It also allows speculation on the 
JASA

possible enhancements that the time-varying nature of the call during feeding could give to the whale in this mechanism for the bubble net feeding by humpback whales.

${ }^{a}$ Also at: Key Laboratory of Marine Information Acquisition and Security (Harbin Engineering University), Ministry of Industry and Information Technology, Harbin, 150001, China.

b) Also at: Key Laboratory of Marine Information Acquisition and Security (Harbin Engineering University), Ministry of Industry and Information Technology, Harbin, 150001, China. Electronic mail: liusongzuo@hotmail.com. 


\section{INTRODUCTION}

The humpback whale (Megaptera noraeangliae) is a baleen whale, known for its unique song and extraordinary feeding behaviour. In particular there is a complex behaviour, bubble net feeding ${ }^{1,2}$, as shown in Fig. 1 (a) and (b). A major prey item for humpback nets at the time is the Atlantic herring (Clupea harengus pallasi) ${ }^{2}$. As described by Wiley et $a l .{ }^{3}$, humpback whales produce a bubble cloud in the shape of upward spiral or double loop around $20 \mathrm{~m}$ below the surface. A number of research investigations ${ }^{2-7}$ have suggested that the bubble cloud could serve as a trap to manipulate herring schools to gather at the center of the relatively bubble-free region. In spite of this, there was no convincing explanation for why the prey should avoid crossing the bubble wall, since undersea bubbles near the surface are common, and the void fraction is unlikely to be sufficient to cause fish buoyancy problems. In 2004 Leighton $^{4,8}$ published a hypothesis that trapping may occur by manipulating the sound of the whale feeding call in the net to produce a 'wall of sound' deterrent surrounding a 'quiet zone' in which the prey would congregate.

Humpback whales are very vocal balaenoptera. Their songs are highly complex ${ }^{9}$ and they employ a number of social calls ${ }^{10}$. It is reported that humpback whales emit a low-frequency and high intensity feeding call when confining herring schools ${ }^{11}$ in the bubble net ${ }^{5}$. Herring have been shown to change behaviour in response to playback of the humpback whale's feeding call ${ }^{12}$. In 2004, Leighton et al..$^{4,6-8,13}$ proposed a 'wall of sound' theory to explain how prey could be trapped as a result of the relationship between acoustic effects and the bubble net by using the geometric acoustics in a two-dimensional plane. These results 
emphasize the potential trapping effect of feeding calls for herring in bubble nets. The initial modelling used a circular bubble net ${ }^{4,8}$, based on common oral descriptions of the time, but in 2007 Leighton et al. revised this to include a spiral bubble net ${ }^{6,7,13}$ when photographic evidence of this shape became available (Figure 1).

In comparison with most other fish species, herring have acute hearing ${ }^{14,15}$. The upper frequency limit of their hearing range is thought to be $5 \mathrm{kHz}^{15}$. Herring are believed to perceive particle motion and pressure components of a sound field ${ }^{16}$. The vocalizations of humpback whales have been associated with high levels of particle motion ${ }^{17}$. Consequently, it is appropriate to consider the sound field in a bubble in terms of both pressure and particle motion.

In order to predict the acoustic field in the bubble net, Leighton and co-workers ${ }^{4,6,7,13}$ calculated the propagation of sound into and around the bubble net by using ray acoustics, and the attenuating effect of a bubble net to shield the quiet zone within from sound generated outside of the net. This identified the existence of the 'walls of sound' encircling the 'quiet zones' Leighton had predicted in areas appropriate for trapping prey, for both circular $^{4,8}$ and spiral ${ }^{6,13,18}$ bubble nets, using estimates of the sound speed in bubbly water ${ }^{18,19}$. Finite element methods have been widely applied in the fields of acoustic propagation and bioacoustics ${ }^{20}$, and this approach is suitable for examining the case of the humpback whale call within a bubble net of this size.

The objective of this study was to propose a 3D numerical model for the propagation of a whale call within such a net. Considering the importance of particle motion to herring, this paper calculates both the sound pressure and particle velocity in and around the bubble net, 

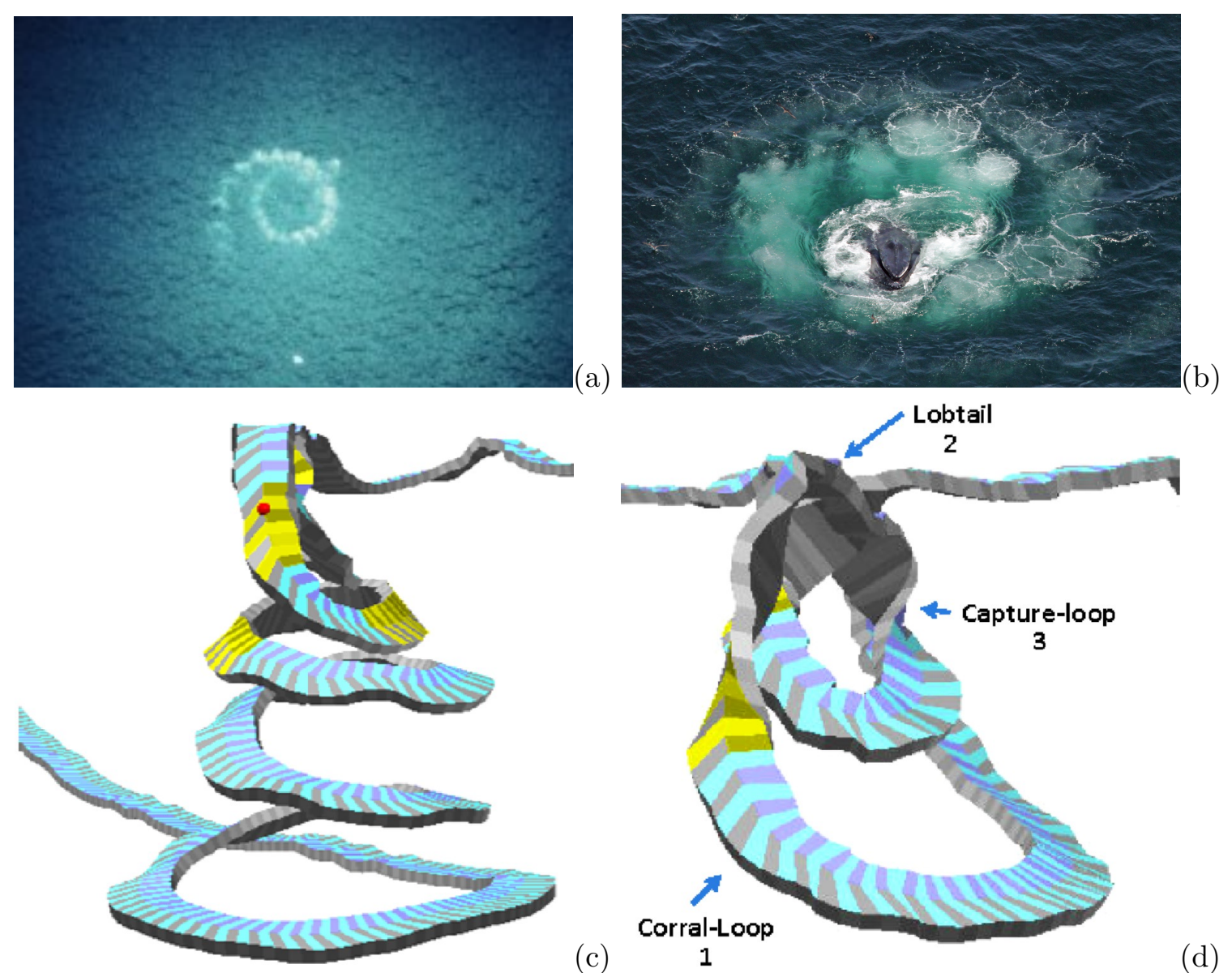

\section{)}

FIG. 1. (Color online) (a) A spiral bubble net created by humpback whales off Cape Fanshaw, Alaska, and photograph by J. Olson, NOAA/NMFS, via Wikimedia commons; (b) A photograph showing the production of spiral bubble net by single humpback whale, and photograph by C. Khan, NOAA/NEFSC, via Wikimedia Commons; The Wiley's DTAG data ${ }^{3}$ of two tracks for bubble net feeding by humpback whales: (c) a upward-spiral net (d) a double-loop net, having permission from Copyright Clearance Center.

respectively. By discussing such plots in the context of the hearing sensitivity of herring, this paper suggests that the distribution of particle velocity in the net may play an important role in trapping herring. 


\section{METHODS AND MATERIALS}

\section{A. Humpback whale feeding call}

In order to formalize the energy distribution associated with the feeding call, a short time Fourier transform was used to represent the multi-component feeding call in the timefrequency domain, as given by

$$
W(t, f)=\int_{-\infty}^{\infty} x(t) w(t-\tau) e^{-j \omega t} \mathrm{~d} t .
$$

The spectrogram of the example feeding call used in this study (available from the electronic supplement) is shown in Fig. 2, whose amplitude is coded by colour. In terms of this spectrogram, as time progresses the feeding call can be divided into three main parts characterized by the pitch at which most of the energy is focused at that time: lower frequency (LF), middle frequency (MF), and higher frequency (HF). The LF component has a modest frequency modulation from 550 to $620 \mathrm{~Hz}$. The MF concentrates energy around $950 \mathrm{~Hz}$, and the burst of HF focuses energy around $1400 \mathrm{~Hz}$. The three crucial frequencies (570, 950 and $1400 \mathrm{~Hz}$ ) were used in simulations.

\section{B. Acoustic propagation in bubbly water}

This section will present the linear mechanism of acoustic propagation in bubbly water that form the basis of the numerical models central to this work. In order to simplify the bubble model, there is a basic assumption that all of bubbles are spherical (departures from bubble sphericity do not, to first order, greatly affect the acoustical calculations, since 


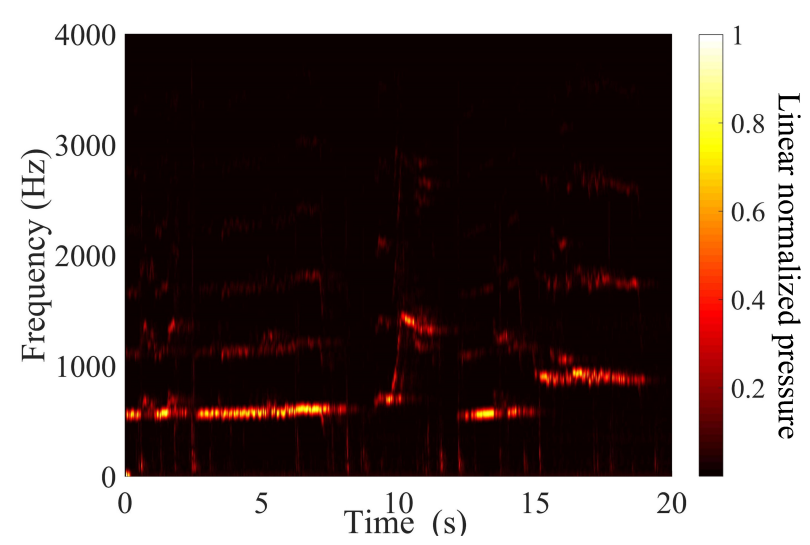

FIG. 2. (Color online) Normalized spectrogram of the feeding calls from humpback whale.

(with the exception of scattering by very large bubbles) they primarily depend on the time variation of the volume of the bubble, not its shape ${ }^{18}$ ). During sound propagation in bubbly water, the interaction between sound and bubbles can be divided into two main actions, volume scattering and radiation from forced oscillation. For circumstances such as this, where the bubbles involved are individually much smaller than the acoustic wavelength ${ }^{21}$, the bubbly water can be represented by an equivalent medium model ${ }^{22}$, and the acoustic propagation in bubbly water can be described by a series of acoustic parameters ${ }^{23}$, which include density $\rho$, sound speed $c$ and attenuation $\alpha$ in units of $\mathrm{dB}$ per meter.

The equation of continuity for bubbly water is given by

$$
\frac{1}{\rho_{w} c_{w}^{2}} \frac{\partial P}{\partial t}+\nabla \cdot \vec{u}=\frac{\partial \beta}{\partial t}
$$

where $P$ and $\vec{u}$ represents the sound pressure and velocity, and $\rho_{w}$ and $c_{w}$ serve as the density and speed of the bubble-free water. Within this equivalent model, the volume fraction $\beta$ can be represented by

$$
\beta=\int n(r) \frac{4}{3} \pi r^{3} d r
$$


where $n(r)$ refers to the number of bubbles per cubic metre per micron increment in bubble radius $r$. It should be noted that there are several basic assumptions for individual bubbles: consistent spherical shape, ideal gas properties within the bubble, uniform pressure distribution within the gas, and consistent bubble distribution throughout the spiral, which of course is unlikely to be the case in the field. Then, the average density of bubbly mixture was calculated as,

$$
\rho_{w}=(1-\beta) \rho_{w}+\beta \rho_{g} .
$$

By using the Van Wijingaarden-Papanicolaou model, the momentum equation of bubbly water can be obtained by

$$
\rho_{m} \frac{\partial \vec{u}}{\partial t}+\nabla P=0
$$

Substituting Eq. (2) into Eq. (3) gives the acoustic propagation equation in the equivalent medium,

$$
\frac{1}{c_{w}^{2}} \frac{\partial^{2} P}{\partial t^{2}}-\nabla^{2} P=\rho_{w} \frac{\partial^{2} \beta}{\partial t^{2}}
$$

Assuming spherically symmetric radial motions and of small amplitude, pulsation is approximately ${ }^{19}$ :

$$
\frac{\mathrm{d} V}{V_{0}}=\left(1+\left(\frac{\mathrm{d} R}{R_{0}}\right)\right)^{3}-1=3\left(\frac{\mathrm{d} R}{R_{0}}\right)+3\left(\frac{\mathrm{d} R}{R_{0}}\right)^{2}+\left(\frac{\mathrm{d} R}{R_{0}}\right)^{3} .
$$

The complex wavenumber ${ }^{22} k_{c}$ within the bubbly mixture can be given

$$
k_{c}^{2} \approx\left(\frac{\omega}{c_{w}}\right)^{2}+4 \pi \omega^{2} \int_{0}^{\infty} \frac{r n(r)}{\omega_{0}^{2}-\omega^{2}+i 2 \beta_{t o t} \omega} \mathrm{d} r,
$$

where the $\beta_{t o t}$ is a dissipation parameter ${ }^{19}$ encompassing of bubble damping by the effects of viscous, thermal and acoustic radiation mechanisms ${ }^{22}$. The parameter $\omega_{0}$ represents the 
bubble's undamped natural frequency. The attenuation coefficient $\alpha$ is given by

$$
\alpha=20 \ln e\left(\frac{\omega v}{c_{w}}\right),
$$

where $\omega$ is the angular frequency driving the sound field, and $v$ serves as the negative imaginary part of sound speed ratio between bubble-free water and the bubbly mixture ${ }^{22}$. The intention here is to represent regions of bubbly water as discrete volumes having frequencydependent sound speeds and attenuation that differ from those of bubble-free water. This is a standard approach, but it is recognized that this is gives an artificially well-defined boundary between the two regions In other regions, the inherent spatial averaging that underpins this form of representation is adequate for lengthscales over which the sound field changes (which indeed can be less than a wavelength if high order modes are excited) but does not attempt to model lengthscales over which one bubble interacts acoustically with its neighbor. These potential issues are alleviated by following the method of Leighton et al. ${ }^{4,6-8}$ of modeling the net as a region where the void fraction is greatest on the center-line of the spiral bubbly arm, and gradually reduces to zero at the point where it meets the bubble-free water. This replaces the well-defined boundary in sound speed and attenuation by a more gradual one, and in practice probably better represents the nets whales produce than would a sudden change in sound speed and attenuation at the edge of the bubble-arm. 


\section{Three-dimensional spiral bubble net modeling}

The bubble net contains two spiral arms, one containing bubbly water, and the other containing relatively bubble-free water that is bounded by its interface with the first (i.e. bubbly) spiral arm ${ }^{6,7,13}$.

Leighton based his original 2004 hypothesis $^{8}$ on photographs of nets from the 1980s that appeared to support the colloquial term 'circle' or 'ring', terms that derive from the dataset up to that time of low-angle surface observation ${ }^{24,25}$, and a limited number of aircraft ${ }^{26}$ observations. However the years after Leighton's 'wall of sound' hypothesis was proposed saw a significant increase in high-quality aerial photographs, which indicated that the description 'spiral' could not be ignored in testing such an acoustical hypothesis, and indeed appeared to be dominant (prior to this, the observation of spiral forms had occurred only as brief passing observations ${ }^{25,26}$.

Leighton conjectured that spirals might be observed in much greater numbers than circles, because they provided specific advantages when forming a 'wall of sound': modeling showed that spiral nets had much greater tolerance for the position of the whale, and could work with only one whale calling whilst the others fed ${ }^{6,7}$, so that spiral nets would present for whales a far easier way to form a 'wall of sound' with an internal quiet region, than would circular nets. These spiral net advantages were summarised in $\operatorname{ref}^{7}$ as follows: "There are however inefficiencies associated with the circular bubble net. To generate a 'wall of sound' (using refraction within the bubbly circle), the insonification needs to be tangential to the walls and, even if it is, the waves which propagate within the bubbly layer are attenuated and 
scattered by the bubbles. Whilst of course sufficient attenuation on its own could generate a 'wall of sound' by simply preventing sound levels within the bubble net from attaining significant values, the refractive component of the 'wall of sound' required both tangential insonification and, if the attenuation were sufficiently great, the sound field might need reinforcing by other whales to generate a complete wall. Furthermore, rays which refract out of the net are effectively wasted energy as they cannot be recaptured by the "wall of sound'. The spiral bubble nets... do not suffer from these disadvantages".

Further weight to Leighton's hypothesis (based on the acoustic advantages deduced in 2007 in $\operatorname{Refs}^{6,7}$ ) that whales might choose to form spiral nets over circular ones was provided in 2011 from tag data of the orientations and movements of whales during bubble netting, obtained by Wiley et al. ${ }^{3}$. They " identified two classes of behaviour (upward-spiral; 6 animals, 118 events and double-loop; 3 animals, 182 events)" ${ }^{3}$. Juarez and Juarez ${ }^{25}$ comparing bubble netting to other forms of fishing by humpback whales during 15,000 hours of observation in Alaska, noted that "Sequences of sounds have been heard only when two whales fed cooperatively using a bubble net to capture herring". Further details on the relative importance of spiral and cylindrical nets are given in the Appendix.

It should be noted that, for acoustical purposes, any visual impressions of the geometry of the bubble net must be treated with caution, especially now that the current paper allows us to extend from 2D to 3D simulations, because with an overall upwards buoyancy force acting alongside turbulence and circulation, bubbles released at depth will tend to rise, and the location of the acoustically important bubbles might not always coincide with the location where they create the greatest visual impact on the sea surface. On the basis of Wiley et 

given in Table $I$

TABLE I. Parameters for spiral bubble net.

\begin{tabular}{cc}
\hline \hline Parameter & Value \\
\hline Number of turns & 2 \\
Maximal spiral radius $(m)$ & 5 \\
Net cross - section radius $(m)$ & 0.7 \\
Axial pitch $(m)$ & 1.5 \\
Radial pitch $(m)$ & -1.5 \\
\hline \hline
\end{tabular}

al.'s description, a simulated bubble net was established by using an upward-spiral geometry for the net's spiral arm of bubbly water as shown in Fig. 3, the associated parameters being

For the upward spiral (Fig. 3), the beginning of the net's spiral arm of bubbly water appears at the outer ring. The term 'Maximal spiral radius', referring to the $10 \mathrm{~m}$ diameter of bubble cloud ${ }^{27}$, is the greatest distance from the vertical axis to the centreline of the spiral. The net cross-section radius is equal to the radius of the vertical cross-section in the net's spiral arm of bubbly water. If the cross-section radius were ever to reach zero, the spiral model would become the center line of the bubble net. The axial and radial pitch respectively determines the vertical and horizontal distance between two points at same normal line but in different turns, and the negative radial pitch refers to how the radius of the net's spiral arm of bubbly water gets smaller as it approaches the air-sea interface. In Fig. 3, 1000 measuring points were vertically placed in the centre of the spiral bubble net, 
and are labeled as $B$. Meanwhile, another 1000 measuring points were placed along the mid-line of the spiral, labeled as $A$.

\section{Finite element simulation for bubble net}

A finite element method (FEM) was applied to simulate the sound field of the humpback whale's feeding call as it propagates through the spiral bubble net, including leakage into the surrounding bubble-free water. This method can be divided into three steps including preprocessing, solver, and result processing. In the first step, a 3D spiral bubble net, modelled following the method of Section C, was placed in the center of the computational domain whose length, width and height are 12.5, 12 and $7 \mathrm{~m}$, as shown in Fig. 3. In terms of the sound source, although the frequencies in the whale's feeding call are generally higher than those of most humpback vocalisations, it was assumed that like their songs ${ }^{28}$, these calls are omnidirectional. Therefore, a point source, which is a pulsating sphere at the limit as the radius tends to zero, was employed as a simulated origin of the humpback whale feeding calls, and was set into the spiral arm of the bubble net at end of the first ring, as shown in perspective view in Fig. 3. Furthermore, in accordance with the measurement from Thompson ${ }^{29}$, the sound source level was set to $169 \mathrm{~dB}$ re $1 \mu \mathrm{Pa}$ at $1 \mathrm{~m}$. In order to eliminate the truncation effect of the boundary, a perfectly matched layer (PML) was applied to replace the hard boundary condition covering the physical region, in order to absorb all the outgoing waves, as shown in Fig. 3.

After building the geometry and boundary conditions, the physics and analytic equations were assigned to different domains by COMSOL Multiphysics. In this model, the water 


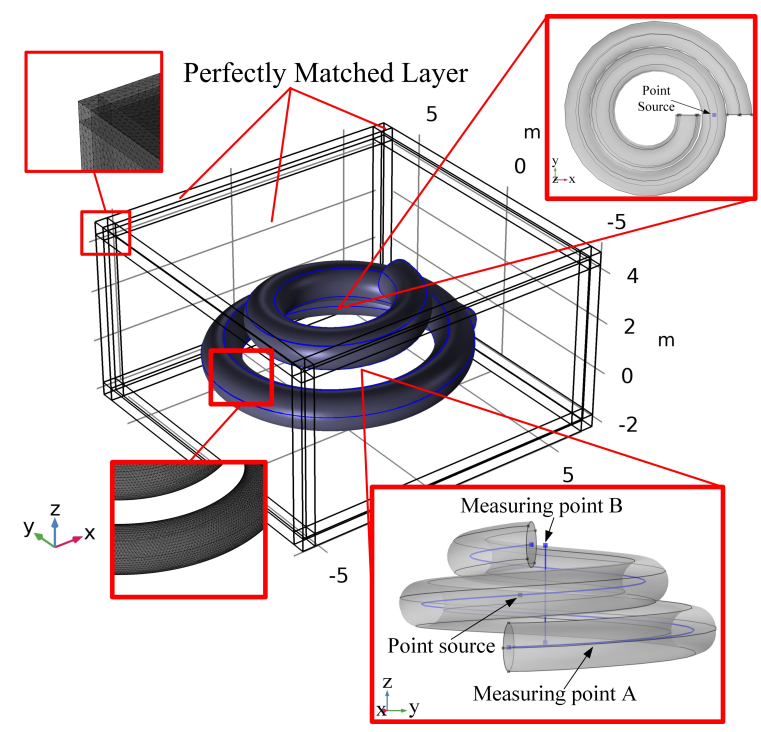

FIG. 3. (Color online) Geometry and mesh for the 3D space including the spiral bubble cloud, the surrounding water and the perfectly matched layer which was used to truncate this model, and the measuring point $\mathrm{A}$ and $\mathrm{B}$ in blue line.

surrounding the bubble net was modeled as a linear elastic fluid, and the sound propagation can be described as

$$
\nabla \cdot\left(-\frac{\nabla P}{\rho_{w}}\right)-\frac{k^{2} P}{\rho_{w}}=0
$$

The bubbly water was assumed to host only linear acoustic propagation, and the sound speed, attenuation and density were calculated by using the equations in Section B. Moreover, the boundary between the bubbly water domain and the surrounding water domain is set to a conditional Dirichlet condition using weak constraints by COMSOL. There are no measurements of the bubble size distribution (BSD) in the whale-generated net to use as input in equation 9. Reidenberg and Laitman ${ }^{30}$ have investigated the production of bubble clouds. They found that humpback whales use their mouths to store air in the oral cavity and whales could then force the air out to release it through the baleen by opening the 
mouth slightly. Reidenberg and Laitman ${ }^{30}$ suggest that this creates many small bubbles in a large volume fraction. Visual impressions of the location, arm continuity and bubbles size can be unreliable for assessing the acoustic effects at high frequencies, because they are skewed to larger bubbles (both because they are more visual and rise more quickly to the surface during bubble netting, ironically creating a greater visual impression because they do not persist in the water subsurface to affect the acoustics). Whilst bubbles much smaller than resonance can reduce the sound speed, those much larger than resonance do not affect the sound speed, having an effect only in the scattering. Bubbles produced in sea water tend to be smaller than those in fresh water ${ }^{31}$, and bubbles generated by injectors that move relative to the flow also tend to be smaller ${ }^{32}$, both because of a reduced tendency to coalesce. Therefore, a proxy constructed from at-sea data, produced by a dynamic injector, was found in the form of the BSD generated by ocean waves in the surf zone, as measured by Leighton et al. ${ }^{19}$. As shown in Fig. 4, this distribution, having bubble radii ranging from 5 to 140 $\mu \mathrm{m}$ with peak radius $19 \mu \mathrm{m}$, could be a reference for the small bubbles size distribution at a volume fraction $1.321 \times 10^{-4}$, and the parameters of the single bubble in spiral arm of the bubble net are shown in the Table $I I$. Under breaking waves, bubbles reach significant depths when circulation and turbulence triumph over the buoyancy forces on that bubble. The causes a sorting effect that makes it easier for smaller bubbles to reach depth than larger ones $^{33}$ whereas in bubble netting all of the gas starts at depth when released by the whale. It might therefore be thought better to use data from seabed seeps as the proxy. However in seeps the injector is stationary, giving rise to bubbles that tend to be larger as a result of coalescence $^{34}$. Therefore for future studies an improved BSD could be found by acoustically 
inverting (using the methods of Leighton and White ${ }^{35-37}$ ) the sounds of the bubbles being released by the whales as recorded by the tags used in experiments like that conducted by Wiley et al. ${ }^{3}$, in order to determine the BSD and void fraction in the net. This challenge is currently beyond the scope of this paper.

In this bubbly mixture, therefore, the density, phase velocity and attenuation distribution was calculated as a function of frequency and volume fraction by solving Eq. $(4,8,9)$. Furthermore, the bubble volume fraction is assumed to linearly decrease with the increasing distance from the center of the bubble cloud that forms the bubbly arm of the spiral bubble net. No account is made for the increase in general bubble size as bubbles rise: whilst (in the absence of dissolution) an isothermal model will reflect a change in void fraction (which will increase by a factor of 2 from the base of the net at $10 \mathrm{~m}$ depth to the surface, a journey that for the small bubbles that affect the sound speed will not be completed until minutes after the feeding), it will not include the effects of fragmentation and coalescence.

Before solving a finite element problem, efficiently meshing can reduce the computational requirements while providing accurate solution. The model, shown in Fig. 3, was automatically divided into smaller finite elements by using free meshed tetrahedral elements which can more easily fit complex geometry. The meshing criterion ${ }^{38}$ uses six elements per wavelength, applied both in the bubble net's spiral arm and the surrounding bubble-free water region, and the corresponding maximum element sizes are 0.089 and $0.179 \mathrm{~m}$. In the second step of the finite element analysis, a fully coupled method was applied to obtain the solution of the sound field in the bubble net's spiral arm of bubbly water and in the surrounding bubble-free water using the COMSOL Multiphysics software. A stationary solver was used 
to find the solution to the steady-state sound field with 6,300,000 degrees of freedom, such that the 3D simulation demands 63 GB of physical memory with a memory allocation factor of 1.2 .

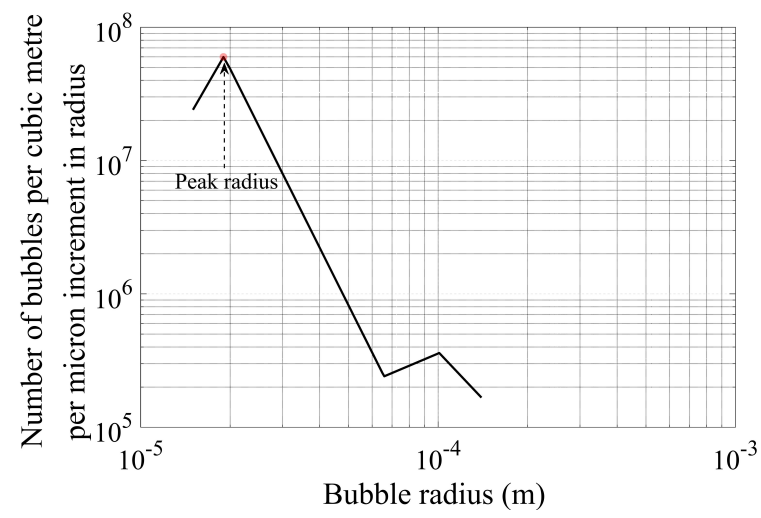

FIG. 4. (Color online) A bubble distribution function taken from an at-sea measurement by Leighton et al. ${ }^{19}$.

\section{RESULTS}

The acoustic propagation parameters were calculated using linear acoustic theory in bubbly water, and the attenuation, phase velocity and density are shown in Fig. 5, for different volume fractions and different frequencies, whose range covers the frequency content of feeding calls and harmonic components from 0 to $5 \mathrm{kHz}$. As the volume fraction (maximum volume fraction: $1.321 \times 10^{-4}$ ) decreases, these three parameters gradually approach the value of bubble-free water, and there is a significant gradient of sound speed, approximately from 780 to $1500 \mathrm{~m} / \mathrm{s}$. It should be noted that, as the stimulated frequency increases to- 
TABLE II. Parameters of the single bubble in spiral arm of the bubble net

\begin{tabular}{cc}
\hline \hline Parameter & Value \\
\hline Density of water $\left(\mathrm{kg} / \mathrm{m}^{3}\right)$ & 998.2 \\
Density of air $\left(\mathrm{kg} / \mathrm{m}^{3}\right)$ & 1.17 \\
Sound speed of water $(\mathrm{m} / \mathrm{s})$ & 1500 \\
Dynamic viscosity $(\mathrm{Pa} \cdot \mathrm{s})$ & $1.0042 \times 10^{-3}$ \\
Surface tension $(\mathrm{N} / \mathrm{m})$ & $72 \times 10^{-3}$ \\
Thermal diffusivity of the $\mathrm{gas}\left(\mathrm{m}^{2} / \mathrm{s}\right)$ & $2.5 \times 10^{-5}$ \\
Polytropic constant & 1.4 \\
Hydrostatic pressure $(\mathrm{Pa})$ & $1.01 \times 10^{5}$ \\
\hline \hline
\end{tabular}

wards the maximum values considered here, the attenuation rapidly rises, and there are slight variations in phase velocity, but the density of the bubbly mixture remains the same.
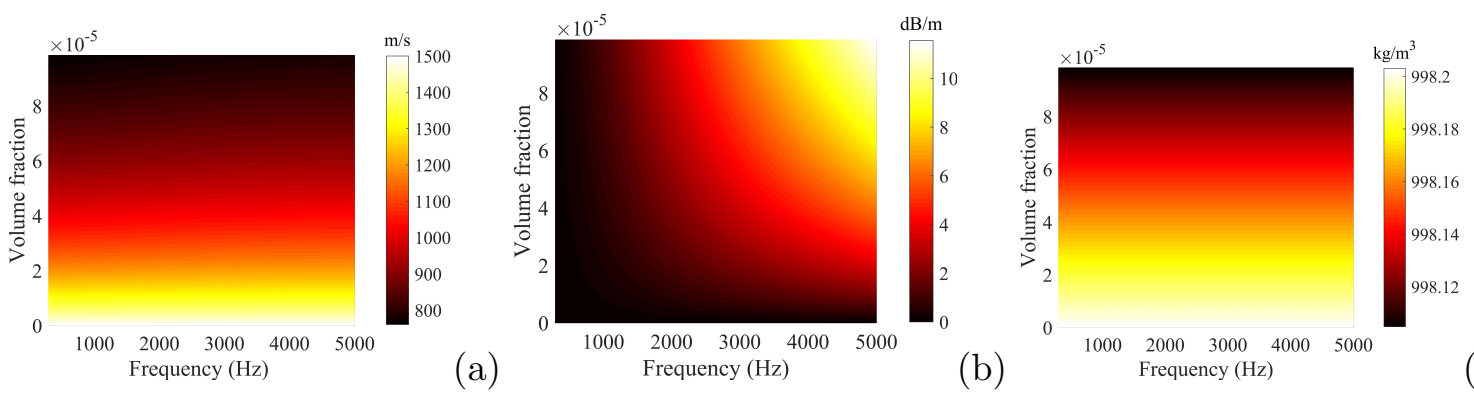

(c)

FIG. 5. (Color online) The volume fraction and frequency dependencies of (a) phase velocity (m/s),

(b) attenuation $(\mathrm{dB} / \mathrm{m})$ and $(\mathrm{c})$ density $\left(\mathrm{kg} / \mathrm{m}^{3}\right)$ in bubbly mixture. 

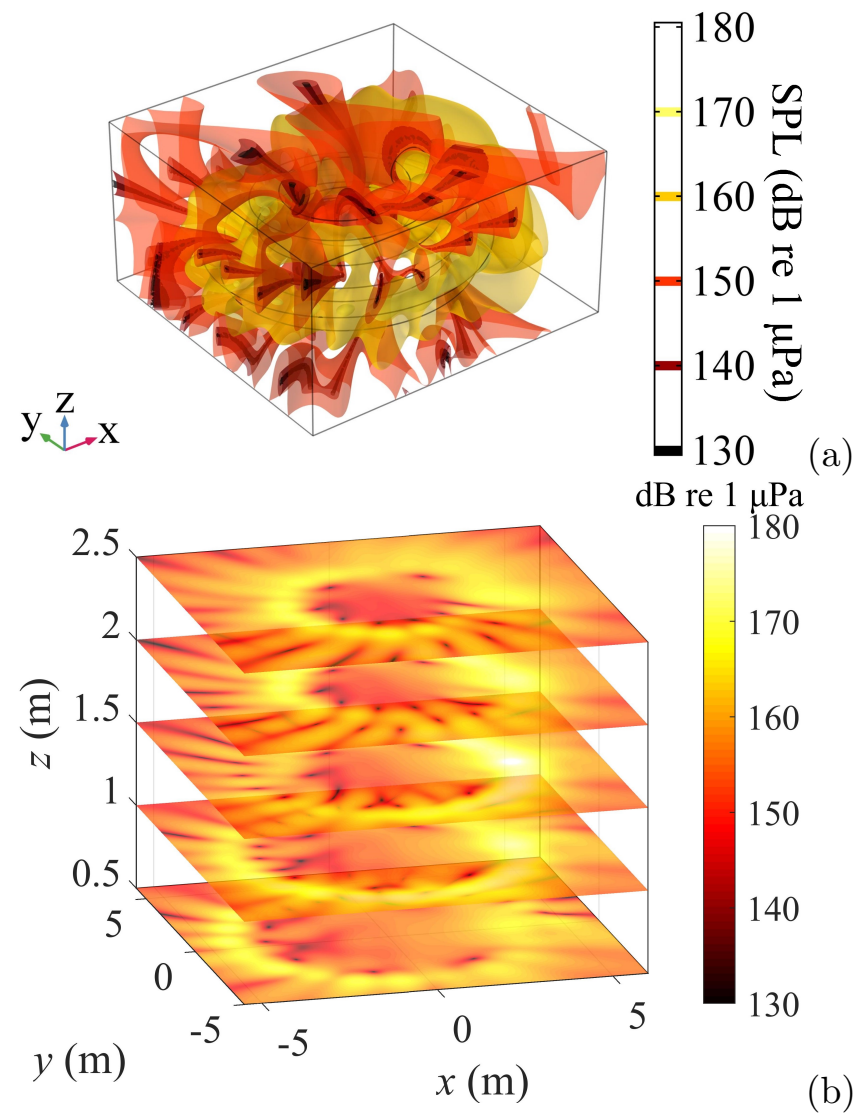

FIG. 6. (Color online) At $570 \mathrm{~Hz}$ (a) sound pressure level of isosurface in the solution domain; (b) sound pressure level of horizontal section at $z=0.5, z=1, z=1.5, z=2$, and $z=2.5$.

A series of numerical results were obtained from finite element simulations, based on the above acoustic propagation parameters. From the $570 \mathrm{~Hz}$ solution, the sound pressure level (SPL) of the total solving field was calculated, and is shown in Fig. 6 and the SPL is represented by six isosurfaces which are made up of points with a constant SPL in a 3D surface. An orange isosurface (the third highest level) covers the bubble net field, and it means that the minimum SPL in the bubble net is approximately $160 \mathrm{~dB}$ re $1 \mu \mathrm{Pa}$. Meanwhile, the primary isosurface is $150 \mathrm{~dB}$ re $1 \mu \mathrm{Pa}$ outside the spiral arm of the bubble net. Therefore, there is at least $10 \mathrm{~dB}$ SPL difference between the wall (which throughout 
this paper refers to the bubbly spiral) and interior (which throughout this paper refers to the bubble-free water encircled by the spiral bubbly wall) of the bubble net. In order to further investigate the sound pressure distribution, the three dimensional space was sliced into five $x-y$ planes at different depth, and are shown in the Fig. 6 (b). The point source located in the plane for which $z=1.5 \mathrm{~m}$ has the the strongest SPL. In bubbly water, the SPL gradually attenuated as the propagating distance increased (where the SPL varies between 160 and $183 \mathrm{~dB}$ re $1 \mu \mathrm{Pa}$ ). Modal behavior, and an $e^{i m \theta}$ radiation (where $m$ and $\theta$ are the azimuthal mode number and coordinate respectively), are clearly present, and could be further explored by an analytical investigation, adapting the approach for cylindrical geometries ${ }^{39,40}$ but using curvilinear coordinates. There are two movies in the supplementary materials that better display the isosurfaces ${ }^{41}$ and horizontal cross-sections ${ }^{42}$ through the sound pressure distribution.
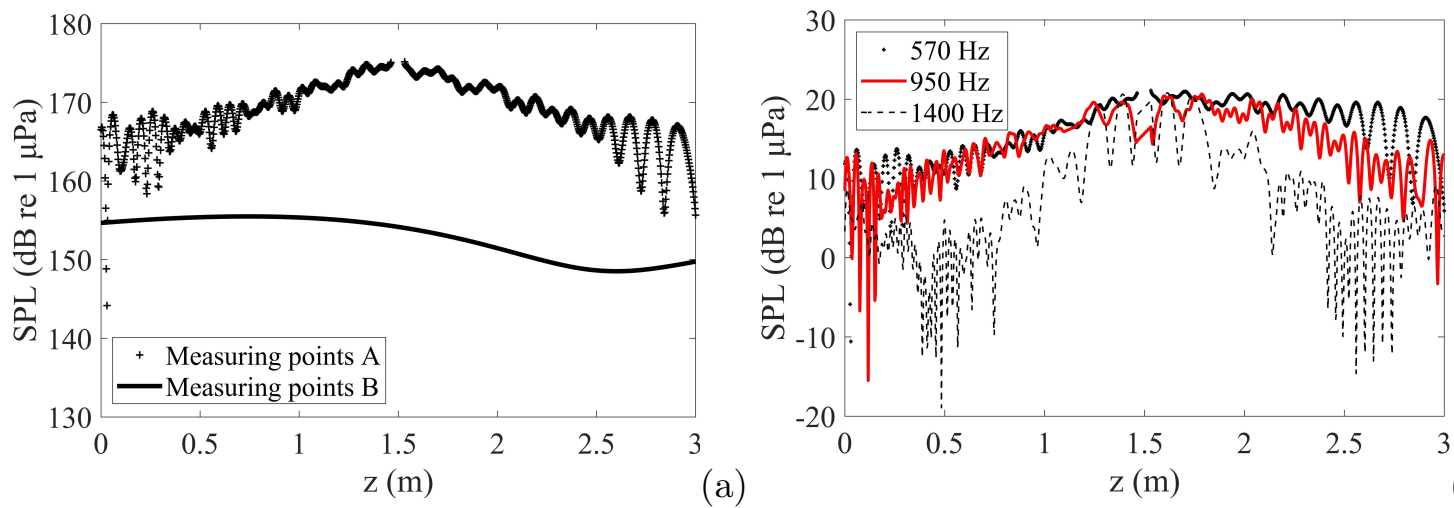

FIG. 7. (Color online) (a) SPL at example measuring points typifying the interior (at point B) and wall (at point A) of the bubble net observed at measuring points A and B; (b) The difference of SPL between measuring points $\mathrm{A}$ and $\mathrm{B}$ at frequencies $570 \mathrm{~Hz}$ (black plus sign), $950 \mathrm{~Hz}$ (red line) and $1400 \mathrm{~Hz}$ (black dotted line). 
Two series of measuring points, A and B, were employed to obtain the SPL at example measurement locations in the interior encircled by the wall, and in the bubbly wall itself, and are shown in Fig. 7 (a). It should be noted that the point source was located at measuring point $\mathrm{A}$ at $z=1.5 \mathrm{~m}$, and an impulse function was applied to form that point source in the finite element simulation. Therefore we removed 20 points around the point source to eliminate the effect of the singular sound function. As sound propagates away from the source, the SPL gradually attenuates inside the spiral arm of the bubble net. The attenuation increases as the frequency increases. There is an evident SPL difference between measuring points $\mathrm{A}$ and $\mathrm{B}$ at the same value of $z$. In order to quantify the SPL differences between the interior (at point B) and wall (at point A) of the bubble net, the differences between measuring points A and B were respectively obtained at $570 \mathrm{~Hz}, 950 \mathrm{~Hz}$ and 1400 $\mathrm{Hz}$, as shown in Fig. 7 (b). There is significant attenuation in the high frequency case, especially in the case of $1400 \mathrm{~Hz}$. Sources placed at most positions outside of the net would generate even larger differences because of the additional attenuation.

As a crucial component of sound waves in water, the particle velocity was calculated for measurement points typifying the interior (at point B) and wall (at point A) of the spiral arm of the bubble net, and is displayed in Fig. 8. In Fig. 8 (a), the particle velocity level (PVL) is represented by five three-dimensional isosurfaces. The isosurface for $40 \mathrm{~dB}$ re 1 $\mu \mathrm{m} / \mathrm{s}$ PVL, covers the bubble net space. It shows that the minimum of PVL in the bubblefree interior of the bubble net is approximately $40 \mathrm{~dB}$ re $1 \mu \mathrm{m} / \mathrm{s}$. In comparison with the isosurfaces in the bubble-free interior of the bubble net, there are some scattered isosurfaces at $30 \mathrm{~dB}$ re $1 \mu \mathrm{m} / \mathrm{s}$ within the bubbly spiral arm of the bubble net. This means that the 

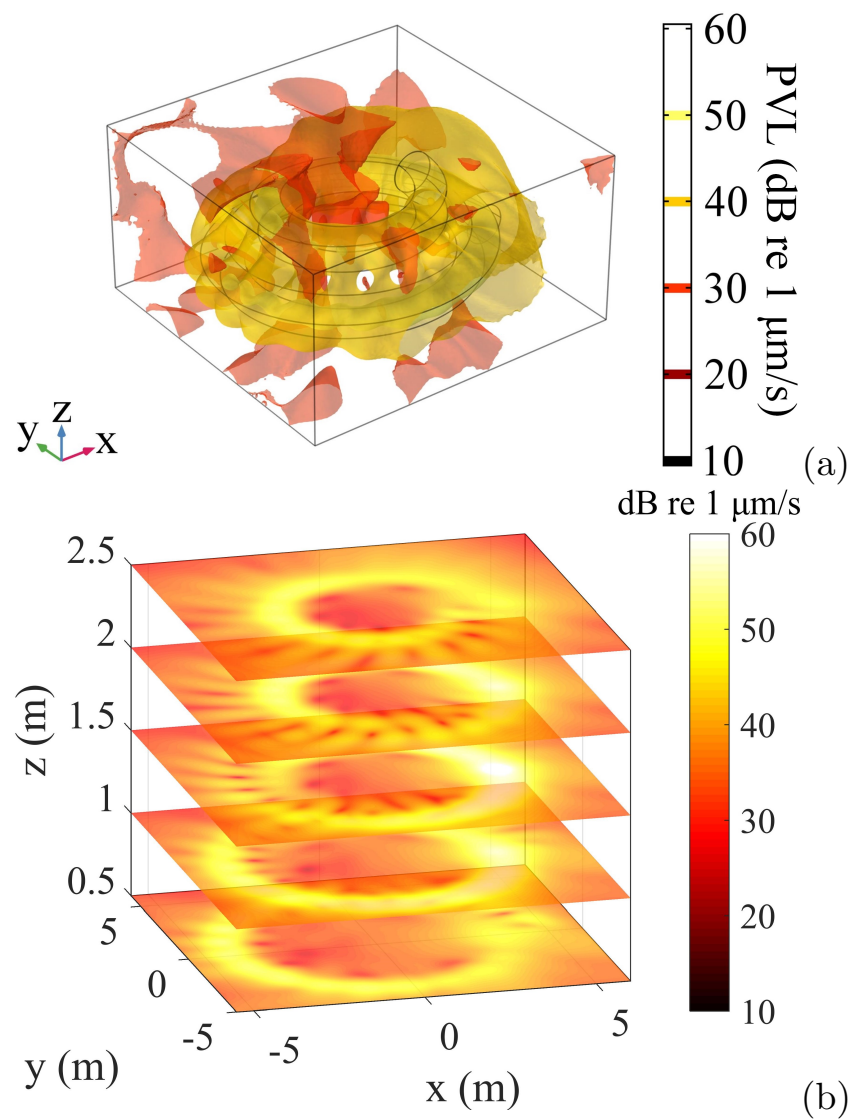

FIG. 8. (Color online) At $570 \mathrm{~Hz}$ (a) particle velocity level of isosurface in the solution domain; (b) particle velocity level of horizontal section at $z=0.5, z=1, z=1.5, z=2$, and $z=2.5$.

outside PVL is in the range of 20 to $30 \mathrm{~dB}$ re $1 \mu \mathrm{m} / \mathrm{s}$. Thus, there is at least $10 \mathrm{~dB}$ PVL difference between the interior (encircled by the wall) and the bubbly wall of the bubble net. Five horizontal $x-y$ planes were applied to slice the three dimensional space for further investigation of the PVL distribution, and the result is shown in Fig. 8 (b). The particle motion field of the bubble net is clearly distinguished in these five planes, because of a strong PVL region in the bubbly arm of the spiral net, and the PVL is $20 \mathrm{~dB}$ less in the centre of the spiral net. 

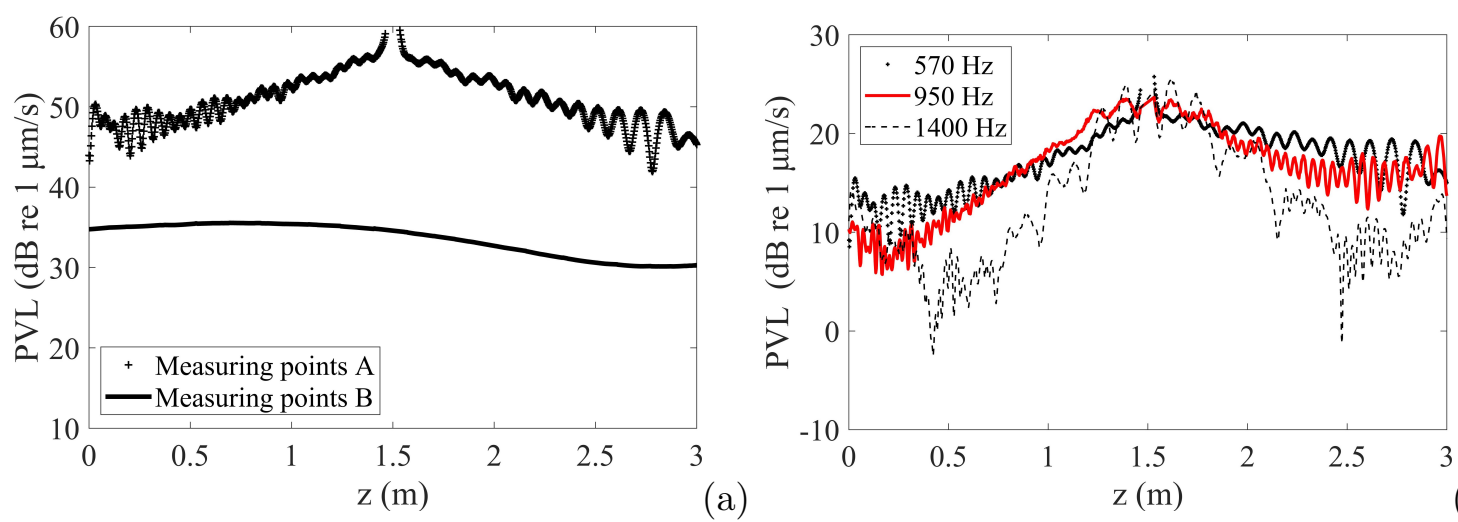

FIG. 9. (Color online) (a) Particle velocity level for inside and outside of bubble net observed in measuring points A and B; (b) The difference of particle velocity level between measuring points $\mathrm{A}$ and $\mathrm{B}$ at frequencies $570 \mathrm{~Hz}$ (black plus sign), $950 \mathrm{~Hz}$ (red line) and $1400 \mathrm{~Hz}$ (black dotted line).

To further examine the particle motion field, the PVL was calculated at the measuring points typifying the interior (at points B) and wall (at points A) of the bubble net by using a corresponding series of measuring points A and B, as shown in Fig. 9 (a). When sound was emitted from the source, the PVL was gradually attenuated as the sound propagated inside the spiral arm of the bubble net. In Fig. 9 (b), the lower frequency component can effectively generate a PVL difference between the example measuring points typifying the interior (at point B) and wall (at point A) of the of bubble net, especially at $570 \mathrm{~Hz}$. It should be noted that there is stronger particle motion at a specific depth (sound source depth) which is from $z=1.36$ to $1.64 \mathrm{~m}$, at $1400 \mathrm{~Hz}$. As a result, it can be seen that the particle motion distribution is frequency dependent. The LF component can contribute to the global PVL in the bubble-free interior of the bubble net. In contrast, the HF component produces high intensity particle motion at a specific depth (the depth of sound source). 

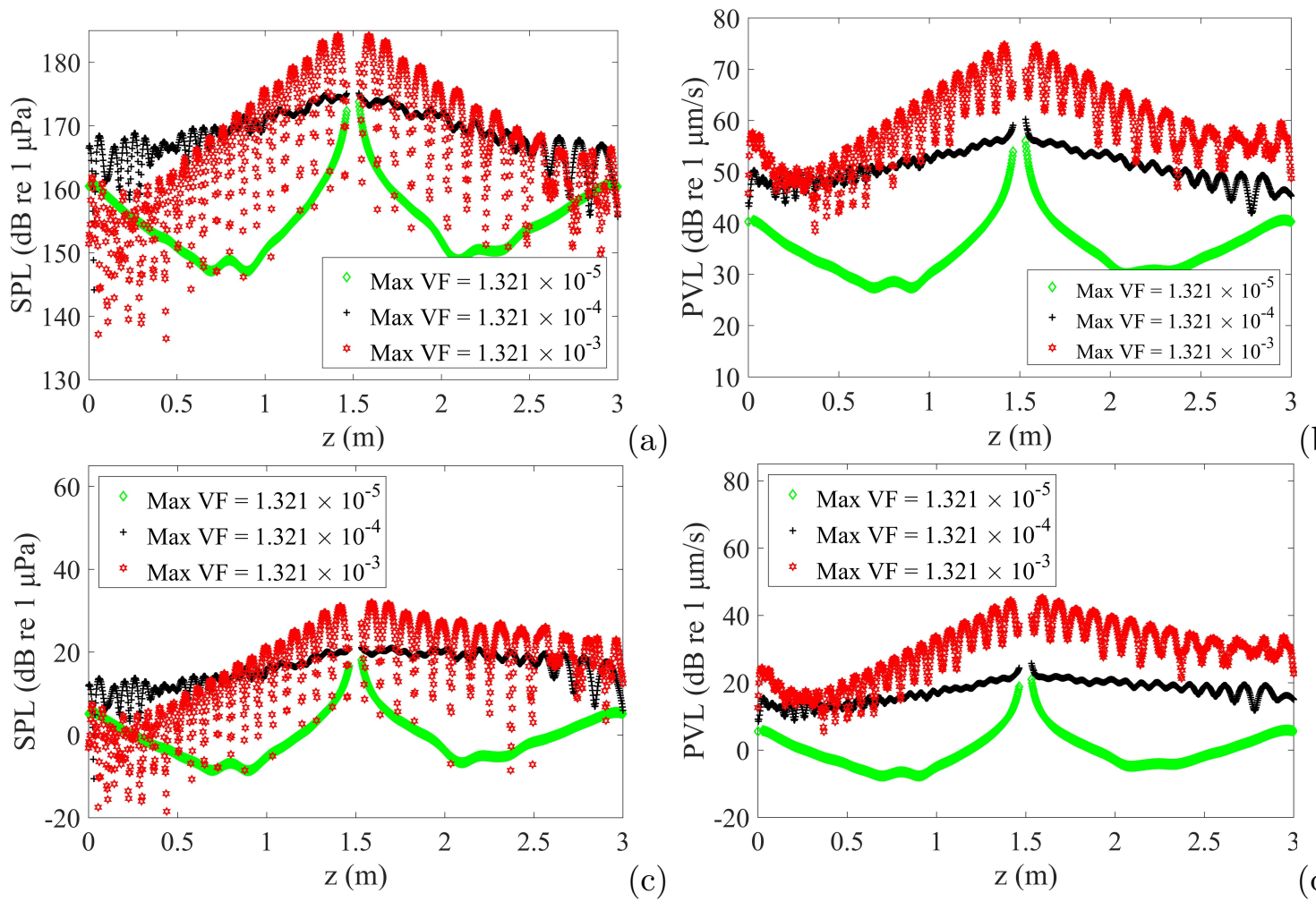

FIG. 10. (Color online) (a) The SPL, (b) the PVL, (c) the difference of SPL, and (d) the difference of PVL, for the three bubble nets with maximal volume fraction $=1.321 \times 10^{-5}$ (green diamond), $1.321 \times 10^{-4}$ (black plus sign) and $1.321 \times 10^{-3}$ (red hexagrams) as centerline of the bubbly arm.

The BSD (Fig. 4) with the maximum volume fraction (VF) $1.321 \times 10^{-4}$ as centerline of the bubbly arm, is scaled to generate three bubble nets with maximum VF $1.321 \times 10^{-5}$, $1.321 \times 10^{-4}$ and $1.321 \times 10^{-3}$ in the bubbly arm, by proportional changing the number of bubbles. SPL and PVL in the bubble net arm are observed in measuring points A for the three cases with different maximum VF, and are respectively shown in Fig. 10 (a) and (b). Furthermore, at the same depth, the differences in SPL and PVL between the bubbly arm and the bubble-free region are obtained at measuring points $\mathrm{A}$ and $\mathrm{B}$ for the three bubble nets with different max VF, as shown in Fig. 10 (c) and (d). Fig. 10 (a) indicates 
that the SPL attenuation rate of higher VF is faster than the case of lower VF in bubble net. However, there is a sound pressure field with the strongest SPL for the case of VF $1.321 \times 10^{-3}$ from $z=0.74$ to $2.57 \mathrm{~m}$. It should be noted that there is an unusual trend for the case of VF $1.321 \times 10^{-5}$ : the SPL rapidly attenuating as the vertical distance between measuring point and sound source increases, but the SPL starts to rebound when the SPL achieves the local minimum value at $z=0.895$ and $2.096 \mathrm{~m}$. In Fig. 10 (b), for particle motion, a comparison of three cases in different VF reveals that the bubble net in higher VF produces stronger particle motion. In contrast to the cases of VF $1.321 \times 10^{-3}, 1.321 \times 10^{-4}$, there is a valley value for the case of VF $1.321 \times 10^{-5}$ at $z=0.900$ and $2.084 \mathrm{~m}$. In Fig. 10 (c), the difference of SPL rapidly decays until it reaches the minimum (less than $0 \mathrm{~dB}$ ) at $z=0.895 \mathrm{~m}$ for the case of $\max \mathrm{VF}=1.321 \times 10^{-5}$, and a similiar finding also appears in the case of PVL as shown in Fig. 10 (d). The comparison of the SPL and PVL difference shows that the bubble net with larger max VF can form a bigger difference of SPL and PVL between bubbly arm and bubble-free water.

To examine the effect of bubble size, Figure 11 (a) and (b) display the SPL and PVL for the bubble nets, whose BSDs have four cases of single bubble size $r=1.9,19,190,1900$ $\mu \mathrm{m}$ and one mixed bubble size (Fig. 4). Moreover, at the same depth, the differences of SPL and PVL between the bubbly arm and bubble-free region are obtained by measuring the signal at the points $\mathrm{A}$ and $\mathrm{B}$ for the five bubble nets with different BSDs, as shown in Fig. 11 (c) and (d). To avoid the influence of VF, the VF of four distributions with single bubble size are consistent with the case of mixed BSD. As the vertical distance to sound source increases, the SPL and PVL both gradually decrease as shown in Fig. 11 (a) and 

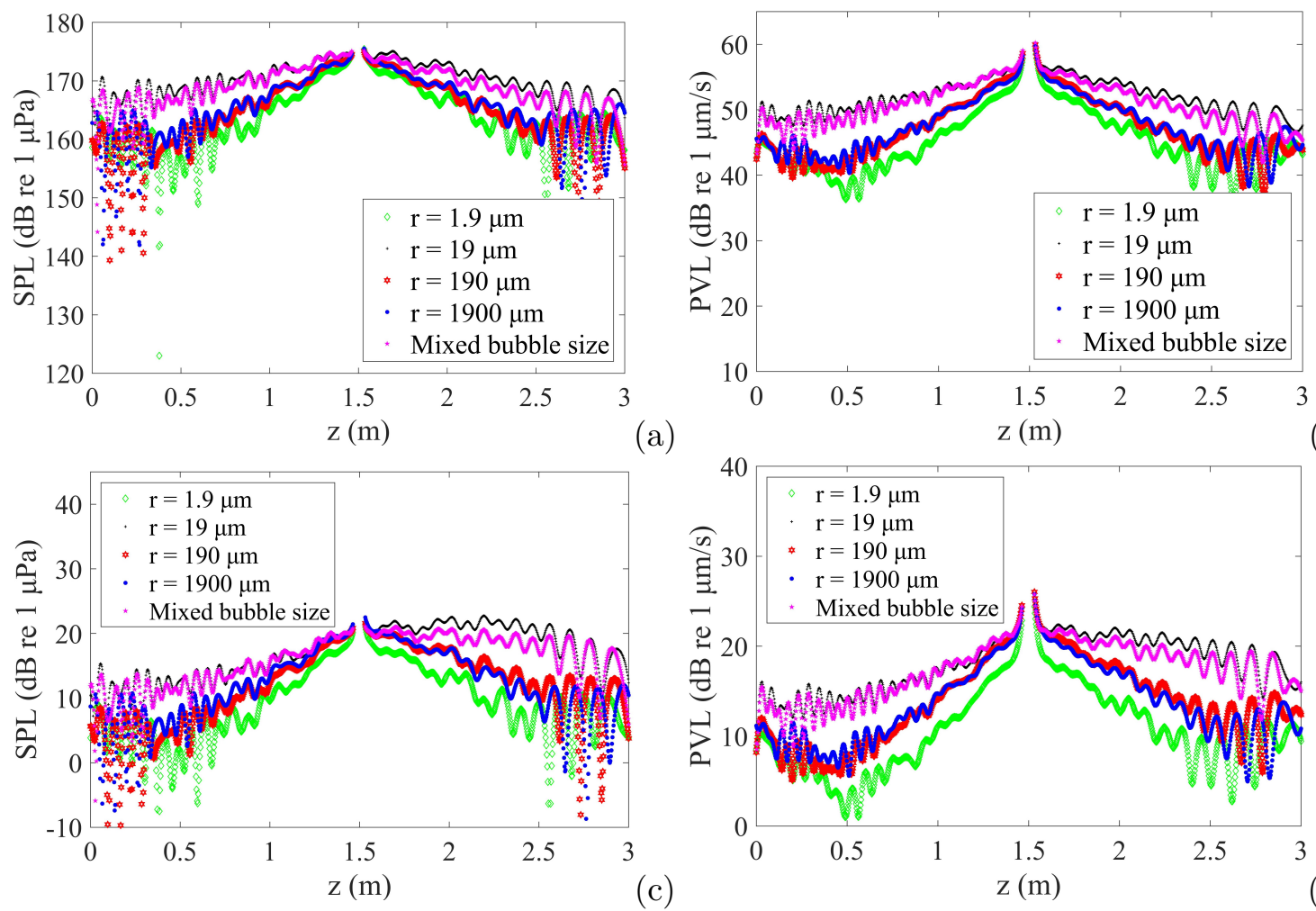

(c)

FIG. 11. (Color online) (a) SPL, (b) PVL, (c) the difference of SPL, and (d) the difference of PVL for the bubble nets with different BSD, including the BSD with single bubble radius $=1.9 \mu \mathrm{m}$ (green diamond), $19 \mu \mathrm{m}$ (black plus sign), $190 \mu \mathrm{m}$ (red hexagram) and $1900 \mu \mathrm{m}$ (blue asterisk), and a BSD with mixed bubble radius (pink pentagram).

(b). The curve for the mixed BSD is in substantial agreement with the case of a $19 \mu \mathrm{m}$ bubble radius, from the view of both sound pressure and particle motion. By comparing with the cases of single bubble size, moreover, the case of $19 \mu \mathrm{m}$ bubble radius produces the strongest sound field, and the largest difference of sound field between the bubbly arm and the bubble-free region. 


\section{DISCUSSIONS}

Previous studies have shown that humpback whales employ bubble nets to prey on herring schools. Herring can detect the feeding calls of humpback whales ${ }^{12}$. Leighton ${ }^{8}$ suggested $^{2}$ the relation between bubble nets and the acoustic effect ${ }^{4,8}$ explored here, with his team using ray acoustic models for a circular bubble net, later revising their $2 \mathrm{D}$ modelling to accommodate a spiral bubble net ${ }^{6,7,13}$. This paper extends that investigation to 3D models. Owing to the high frequency approximation inherent in the ray acoustics model, other methods are required to explore lower frequencies, quantify the SPL, and (even though the earlier studies ${ }^{6,7,13}$ introduced the ability of a ray incident on the bubbly water both to scatter energy off it and propagate refracted energy into it) fully explore the interactions that occur as sound in bubble-free water meets the bubbly arm, and vice versa. In this study, we established the three-dimensional upward spiral model to further explain bubble net feeding using a finite element method (FEM), and the simulated model is based on the geometry of the underwater net as described by Wiley et $a l .{ }^{3}$. In terms of the FEM, the accuracy of the simulation has been examined to check that the simulation errors from discretization were insignificant for the meshed size with six elements per wavelength in a previous study ${ }^{38}$

During bubble net feeding, humpback whales have been reported to emit ${ }^{29}$ continuous calls whose sound source level reaches up to $170 \mathrm{~dB}$ re $1 \mu \mathrm{Pa}$ at $1 \mathrm{~m}$. Propagation of the feeding call was affected by the bubbly water, as shown in Fig. 6. Corresponding simulations, in this study, show the details of the propagation pathway inside the bubble net 
and the pressure distribution in the surrounding water ${ }^{6,7}$. In comparison with the pressure distribution of bubble-free water encircled by the spiral, the pressure level is lower than inside the bubbly spiral arm of the net, reducing by $20 \mathrm{~dB}$ towards the quieter, bubble-free interior that the spiral arm encircles as it also encircles prey. These observation hold for a source placed within the bubbly spiral as shown in Figure 3: other source locations will require follow-up investigations, and should be correlated (if possible) to the position of the whale when it calls. In the bubble net simulation, the sound speed monotonously decreases as the VF increases, and the attenuation is proportional to the VF. This gradient of sound speed contributed to a refraction effect in the acoustic propagation pathway, the negative gradient of sound speed controlling how the feeding sound propagates along the spiral centerline of the cloud in the clockwise and anticlockwise directions, respectively. This verifies Leighton's hypothesis of the high SPL are achieved within the walls of the net, and relatively lower SPL in the bubble-free water that is encircled by those arms (Fig. 6 (b)). Owing to the reflection from the interface between the bubbly mixture and the surrounding water, there are interference fringes in the SPL distribution inside the bubble net. Moreover, the results indicate that the attenuation will increase as the frequency increases (Fig. 7(b)). It should be noted that the global attenuation (the acoustic propagation attenuation in this spiral bubble net) is determined by two factors, the attenuation by the bubbly water (the acoustic absorption of the bubbly water, that converts sound ultimately to heat ${ }^{18}$ ) and scattering in the bubbly liquid (where the acoustic energy remains as acoustic energy bubbly mixture). Together with the refraction effect (that causes acoustical focusing or de-focusing, generated by sound speed variations), these factors interact to generate inhomogeneous sound fields 
to produce a "wall of sound" ${ }^{6}$, and this interaction must be a trade-off between acoustic attenuation and focusing. By comparing the difference between measurement points $\mathrm{A}$ and $\mathrm{B}$, the results prove (for the point source positioning and bubble densities assumed here) that an acoustic pressure difference is produced by the interaction between the feeding call and the bubbly cloud, and there is a region with stronger sound field in the spiral arm of the bubble net.

In this study, we calculated the particle velocity level (PVL) to examine the effect of particle motion in bubble net. In Fig. 8 (a), the bubble net can be seen to have generated a three-dimensional surface with intensity particle motion, and this isosurface covers a quiet region with less particle motion. From these results, it is clear that the bubble cloud contributes to controlling the acoustic energy focusing to enhance the particle motion in the spiral arm of the bubble net. Further analysis showed that the bubble cloud produced obvious PVL differences between the bubbly water inside the spiral arm and the bubble-free water encircled by the spiral bubble net, by observing the horizontal cross-sections. Comparison with the PVL of the bubble-free water in center of the spiral bubble net, shows that the PVL inside of the spiral bubble net is over $15 \mathrm{~dB}$ higher. Compared to the results of SPL (Fig. 7), PVL modelling suggests that the particle motion can build a stronger 'acoustic wall'. There is an important finding in the understanding of the spatial variation of PVL at different frequencies (noting of course that the particle velocity depends on the spatial gradient of the acoustic pressure, which can be high for the strong variations in bubble density used in these simulations). Our results demonstrated that there is the largest difference of PVL between measuring points A and B at $1400 \mathrm{~Hz}$ (not unexpectedly, the highest fre- 
quencies and smallest wavelengths tested), at $z=1.35$ to $1.65 \mathrm{~m}$, and this difference rapidly attenuated as the distance (from measuring point to source) increases. In contrast to the case at $1400 \mathrm{~Hz}$, the PVL difference measuring points A and B was maintained over $10 \mathrm{~dB}$ for the case of $570 \mathrm{~Hz}$ signal for all of measuring points, although the maximum is almost $4 \mathrm{~dB}$ lower than the case of $1400 \mathrm{~Hz}$. Therefore, the results imply that these sound components with different frequencies might play distinct roles in the operation of the 'acoustic wall' of the bubble net. The LF components generate a basic 'acoustic wall' covering the bubble net, based on the low-attenuation ability, and then the HF components can further build a stronger 'acoustic wall' at a specific depth (sound source depth) by using the focusing ability of the bubbly arm.

In the bubble net's arm, the sound propagation parameter (sound velocity and attenuation coefficient) are strongly dependent on the BSD (including VF and the bubble radius), and then these factors affect the sound focusing and attenuation in bubbly water. Regarding the BSD produced by humpback whales, there is, unfortunately, no measurement data about the BSD in a bubble net. Considering that is know about the production mechanism for bubbles ${ }^{30}$ (describing in the Section C of Part two), we have conducted simulations by changing the values of the bubble size and the VF to discuss the effect of BSD in bubble net. In terms of the VF, Fig. 10 (a) and (b) indicated that the focusing performance of the bubble net in higher VF is stronger than the case of lower VF by comparing the sound field at $z=0.76$ to $2.45 \mathrm{~m}$. For the case of $\mathrm{VF}=1.321 \times 10^{-5}$, both sound pressure and particle velocity show stronger responses at $z=0,1.5$ and $3 \mathrm{~m}$, the position near to sound source, by reason of the energy leaking from the bubble net. It also should be noted that the 
minimum of the difference of SPL and PVL between the bubbly arm and the bubble-free water, is less than $0 \mathrm{~dB}$ for the case of $\mathrm{VF}=1.321 \times 10^{-5}$. This means that the bubble net with a lower VF (less than $1.321 \times 10^{-5}$ ) is less able to produce an 'acoustic wall' as shown in Fig. 10 (c) and (d). As another essential property of BSD, the bubble radius in bubble net affects the ability of 'acoustic wall', as shown in Fig. 11. The bubble radius controls the natural frequency of the bubbles in bubble net arm, and then it can determine the sound speed, attenuation in bubbly water. Regarding the bubbly water with a fixed $\mathrm{VF}$, the number of bubbles decreases as the bubble size increases. This means that sound velocity is not monotonously changing with bubble size in the model with a invariant VF, referring to Eq. (8). To further study the effect of 'acoustic wall', we observe the strongest sound field in the bubble net arm with $19 \mu \mathrm{m}$ bubble radius when compared with the cases of 1.9, 190 and $1900 \mu \mathrm{m}$. It also should be noted that there is a difference between the case of a mixed bubble size and the case of a single bubble radius equal to $19 \mu \mathrm{m}$ by reason of the bubble distribution function including other bubble components with different radius. Above all, the formation of an 'acoustic wall' in the bubble net is strongly dependent on the bubble size and VF of the BSD. The potential mechanism of bubble net generation ${ }^{30}$, which releases small bubbles to produce a high void fraction, is commensurate with the hypotheses in the earlier papers ${ }^{6,7,13}$ and here, that a 'wall of sound' can be domed surrounding a quiet region to trap prey using attenuation or refraction in the bubbly water, and reflection.

As prey of humpback whales, herring have an acute and sensitive hearing system. Therefore, the characteristics of herring hearing are an important consideration in explaining the trapping performance of the bubble net. In terms of pressure acoustics, the ABR (Auditory 
Brainstem Response) of the herring were obtained by Enger ${ }^{43}$. From this audiogram of herring, the hearing thresholds at $570 \mathrm{~Hz}$ and $950 \mathrm{~Hz}$ are around $100 \mathrm{~dB}$ re $1 \mu \mathrm{Pa}$. For the $\mathrm{HF}$ component at $1400 \mathrm{~Hz}$, the $\mathrm{ABR}$ of herring is $105 \mathrm{~dB}$ re $1 \mu \mathrm{Pa}$, approximately. Given these $\mathrm{ABR}$, it means that the herring hearing is more sensitive to the lower frequencies examined in this paper, and that the feeding sound of humpback whales overlaps with the sensitive hearing range of herring. Considering the effect of bubble net, the SPL inside the bubbly arms of the spiral, ranged from 160 to $175 \mathrm{~dB}$ re $1 \mu \mathrm{Pa}$, is at least $60 \mathrm{~dB}$ higher than the auditory brainstem threshold of herring at $570 \mathrm{~Hz}$. Furthermore, it has been verified that herring can distinguish feeding calls of humpback whales from observing their avoidance behavior. Sharpe ${ }^{12}$ replayed feeding calls of humpback whales, whose sound pressure level is ranged from 130 to $135 \mathrm{~dB}$ re $1 \mu \mathrm{Pa}$, to examine the herring response by using the playback experiments, and then he found herring readily perceived feeding calls and moved away from the speaker. Meanwhile, Rieucau ${ }^{44}$ found that the herring school exhibited a stronger collective escape behavior, owing to a vocal behavior of killer whales. From the hearing sensitivity of herring, Handegard ${ }^{45}$ suggests that fish (including herring) can produce different behavioural responses for the subtle changes in sounds. These observations tally with the proposition $^{6,7}$ that herring could avoid the strong sound field, and herring would gather in the relatively quiet region in middle of the spiral net to avoid the loud feeding call. The SPL distribution in the bubble-free water at the vertical centreline of the net would be perceived by herring. But there is such a strong field, whose SPL is at least $60 \mathrm{~dB}$ higher than herring ABR, of acoustic pressure in the bubbly spiral arm surrounding the bubble-free region that herring will likely find this region to be a deterrent. Thus, it suggests that the acoustic 
effect in the bubble cloud could generate a 'wall of sound' ${ }^{4}$ to control herring trapping in the bubble net.

Leighton $^{6-8}$ included in his hypothesis that quiet zones and loud walls in the bubble nets of humpback whales could not only interact with hearing systems, producing avoidance and startle behaviours, but also excite swim bladder resonances. In the context of particle motion $^{46}$, the swimbladder plays a significant role as a herring auditory organ. Popper et al. ${ }^{15}$ suggested that herring have a complex hearing system, which includes swimbladder and inner ear components, for detecting particle motion ${ }^{47}$. As an air-filled organ, the swimbladder can act in a manner which resembles an air bubble. When the swimbladder interacts with an acoustic wave, therefore, it will pulsate and can (at the appropriate frequency) undergo resonance, at which point herring can perceive strong particle motion by swimbladder. The swimbladder of herring was modeled as an elongated spheroidal-shaped linear oscillator ${ }^{48}$ with a swimbladder-resonance correction ${ }^{49}$, and Nero estimated the resonance frequency of the herring swimbladder to be approximately 400 to $600 \mathrm{~Hz}$ at a depth of less than 20 meters. This is consistent with the frequency range of the LF component in humpback feeding calls, and it means that the feeding sound of the humpback whale can stimulate the swimbladder resonance. From the PVL distribution, shown in Fig. 9, there is a $25 \mathrm{~dB}$ (maximum) PVL difference between example measuring points typifying the interior (at point B) and wall (at point A) of the bubble net. Therefore, in order to avoid intense particle motion, herring might be expected to gather towards the bubble-free water in the center of the spiral net, i.e. the 'quiet zone'. These results provide growing evidence that humpback whales could 
apply the acoustic effect of the bubble net to trap herring in the bubble-free water in the center of the bubble net.

We hypothesize that a humpback whale team bubble netting by using the feeding sound shown in Fig. 2, might utilize the various frequency components of the call in the following manner. At the beginning of feeding, the humpback whale uses LF sound to generate an acoustic field to prevent the herring school escaping. Towards the end of the hunt, the whale emits powerful HF components in the sound to focus herring further towards the center of the bubble net, enabling an effective lunge-feed.

There is still, however, a lack of knowledge on the cooperative behavior between humpback whales. In humpback whale populations, group cooperation is extremely common phenomenon $^{5}$, and cooperative behavior might allow humpback whales further to enhance the acoustic effect of the bubble net. Here only the influence of a single whale is considered by use of a single point source. The case of multiple sound sources should be analyzed in future studies, if it is found that multiple whales emit the feeding call during a single hunt. Further, this work has only studied a spiral bubble net, which is the geometry ${ }^{3}$, that current data most supports but other geometries should also be considered as more data is collected and the range of typical geometries elucidated. Finally, further observation of humpback whales' behaviours need to be performed to establish the relationship between the bubble net feeding and acoustic information. It would be important to ascertain whether successful bubble netting occurs without the humpback whales emitting loud calls, to help determine whether the acoustics plays a critical role, or acts as an enhancing adjunct, in the performance of the bubble net. 


\section{CONCLUSION}

This paper has investigated the humpback whale's behaviour of bubble net feeding by using finite element simulation. The FEM has been used to calculate both sound pressure and particle velocity in the spiral bubble net, which is modeled as an equivalent bubbly mixture. The results support Leighton's hypothesis that the bubble net generates louder outer arms, and a relative quieter central bubble-free zone, to which prey might congregate. For the whale's feeding call, there is a significant acoustic difference between the intense sound field produced within the bubbly spiral arms ('wall of sound') and the quieter bubblefree waters that those arms encircle. If the sound source is outside of the net, attenuation will cause the loudest sound there, and the quietest in the net centre. This paper has underlined the importance of particle motion, and it was found that there is a strong particle motion field inside the bubbly spiral arms. In terms of the relavant auditory organ, herring are highly sensitive to the feeding call of humpback whales. In the context of the hearing ability of herring, the spiral bubble net produced a three-dimensional wall to manipulate the herring school gathering in the bubble-free water which is encircled by the bubble net arms, for both sound pressure and particle motion. Furthermore, the results demonstrated (for the source location and bubble densities chosen) that the sound field was highly frequency dependent, and consequently this paper speculates that humpback whales apply feeding sounds with different frequency components at different feeding stages. In the beginning, low-frequency sound can be used to generate a basic sound field throughout the bubble net for trapping herring schools in the bubble net. At a later stage, high-frequency sound is 
putatively focused in the bubble net at specific heights for gathering herring schools in the center of the bubble net. In conclusion, the evidence from this study supports Leighton's hypothesis that the production of a bubble net by humpback whales produces a spatially inhomogeneous distribution of acoustic energy, notably with the production of quiet zones, capable of trapping prey. The modelling here extends the previous capability to 3D, and produces new insights into the possible advantages that might arise from varying the call frequency during the hunt. These discoveries highlight the need for more observational data from the field on the spatial distribution of acoustic energy in the net, and differences in the behavior of the fish when the sound is present and when it is not.

\section{ACKNOWLEDGMENTS}

This work was conducted when the first author was on a study visit to the University of Southampton, funded by College of Underwater Acoustic Engineering, Harbin Engineering University, and Institute of Sound and Vibration Research, University of Southampton. This work was supported by the National Natural Science Foundation of China under Grants 61771152, National Key R and D Program of China, and the Fundamental Research Funds of the Central University, China Grant No. HEUCFG201825. The authors acknowledge the use of the IRIDIS High Performance Computing Facility, and associated support services at the University of Southampton, in the completion of this work. The data supporting this study are openly available as DOI: [reference to be completed in line with university policy if paper accepted] from the University of Southampton repository at http://dx.doi.org/ [reference to be completed in line with university policy if paper accepted]. 


\section{APPENDIX A: THE EVOLUTION OF THE SPIRAL BUBBLE NET MODEL FROM 2D TO 3D}

In 2004, Leighton et al. ${ }^{4}$ hypothesized that humpback whales might trap prey within a quiet zone surrounded by a 'wall of sound', but modeled the bubble net in the form of $2 \mathrm{D}$ hollow cylinder by humpback whales. Based on the photograph materials in 2007 he adapted the model to employ a $2 \mathrm{D}$ spiral form bubble net ${ }^{6}$, and suggested whales might prefer to make spiral nets instead of circular ones because of their greater ease-of-use for forming a wall-of-sound around a 'quiet zone'. Owing to the vertical movement of humpback whales (rising vertically to the surface ${ }^{50}$ ), however, the 2D model cannot accurately describe the 3D feeding process. In southern Gulf of Maine, Wiley et al. ${ }^{3}$ identified two typical forms bubble net: upward-spiral and double loop. In upward-spiral bubble net feeding, they found that the bubbles are released in a stream throughout the spiral movement path (based on 118 events from six whales). This confirmed Leighton's second hypothesis, that the upwardspiral is a common form for bubble net in $3 \mathrm{D}$ view. The other form of motion seen by Wiley et al., the double-loop bubble net, includes two independent loops in a 3D irregular circle (not in a horizontal plane), and the size of upper loop (capture-loop) is less than that of lower loop (corral-loop). Because the upper loop is the smaller one, it should be noted that if bubbles were to be released continually during a double-loop maneuver, the rising bubbles would produce a form resembling a spiral. Wiley et al. even, noted that some whales generated a spiral-net to achieve a corral-loop in double-loop feeding. This confirms the importance of the spiral geometry in modeling these bubble nets, both because of its 
prevalence and because of the unique acoustical features identified by Leighton, neither of which the circular nets possess. The attractiveness of the circular form derives from the simplicity of axisymmetric modeling, not because it captures either typical whale behavior (based on data to date) or the important acoustical features.

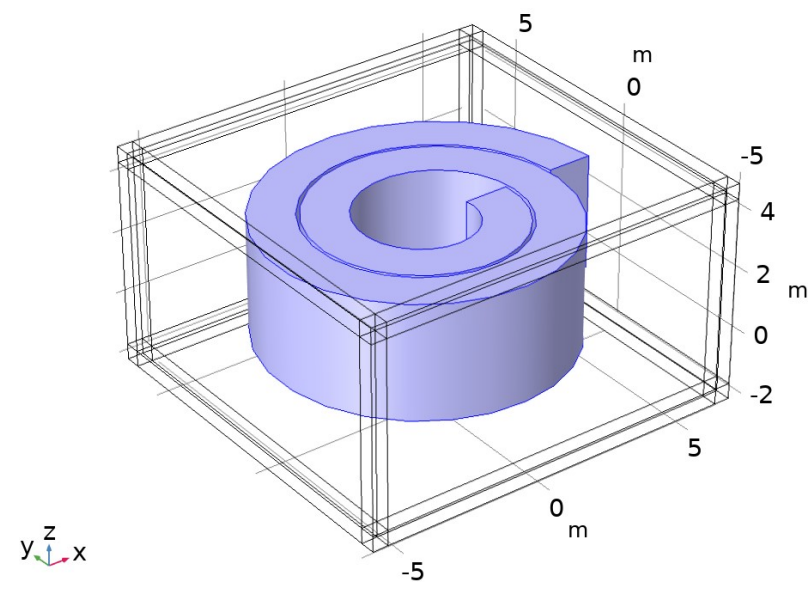

FIG. 12. (Color online) A 3D cylindrical spiral bubble net in a rectangular computational domain which was covered by perfectly matching layer.

In order to bridge the connection between the $2 \mathrm{D}$ model in the previous studies ${ }^{4,6}$ and the 3D spiral bubble net (Fig.3), a 2D spiral (where the bubbly arms are so thick as to touch as they lap one another, eliminating the bubble-free arm of the spiral) has been vertically extended to generate a cylindrical spiral in a 3D space, as shown in Fig. 12, and then this model was solved by the finite element method based on the steps described above.

From the solution at $570 \mathrm{~Hz}$, the 3D sound field have been sliced into five $x-y$ planes at different depth and the SPL and PVL are shown in Fig. 13 (a) and (b), respectively. There are two movies in the supplementary materials to better present the sound pressure ${ }^{51}$ and particle velocity ${ }^{52}$ in a $3 \mathrm{D}$ view. It could be clearly seen that, despite the strong attenuation 


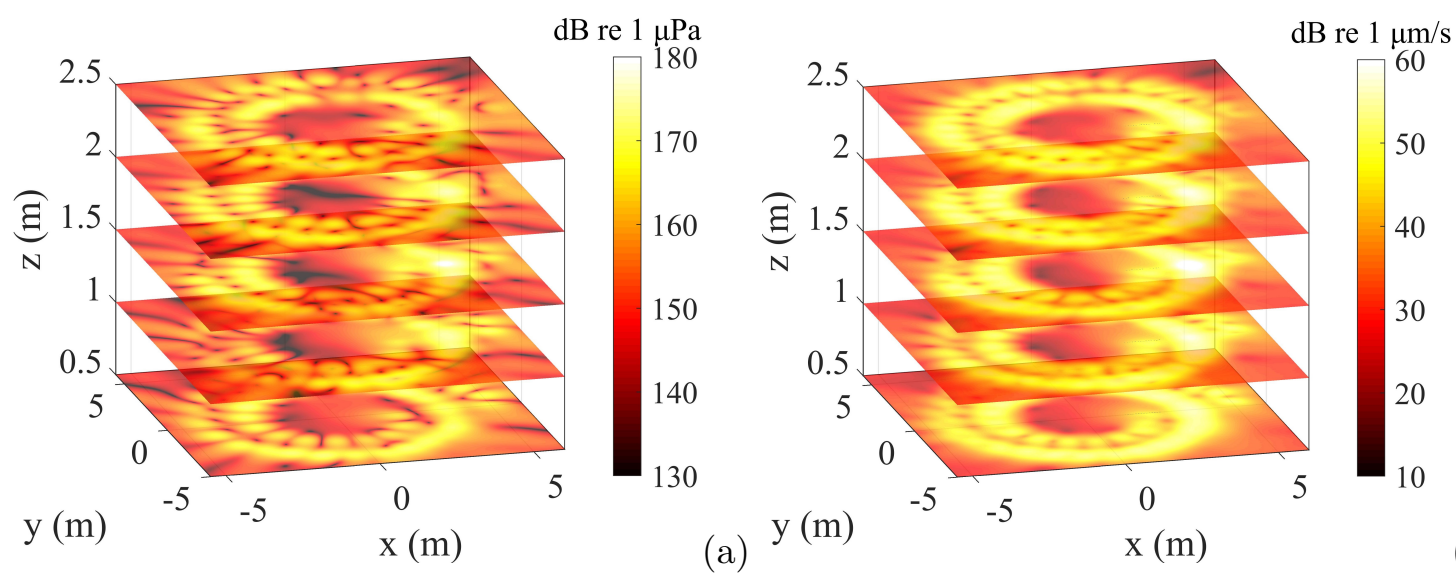

FIG. 13. (Color online) At $570 \mathrm{~Hz}$, (a) SPL and (b) PVL for the horizontal slices of the 3D solution at $z=0.5, z=1, z=1.5, z=2$, and $z=2.5$.

in the bubbly arm which goes up to of $0.4103 \mathrm{~dB}$ per metre at $570 \mathrm{~Hz}$ (the data extracted from the Fig. 5(b)), there is a strong sound field in the arm of bubble net owing to the acoustic focusing effect. In comparison with the sound field in the center of the bubble-free water, the SPL in the arm of the bubble net is at least $20 \mathrm{~dB}$ higher, and the PVL in the arm of bubble net is at least $25 \mathrm{~dB}$ higher. Thus, as a stepping stone, the bubble net in the shape of a 3D cylindrical spiral can form a 'wall of sound' in both sound pressure and particle motion. Comparing with the results of the 3D spiral bubble net (in Fig. 6 (b) and Fig. 8 (b)), the bubble net that has the form of a 3D cylindrical spiral lacks the ability to perform the function ${ }^{3}$ of compacting the herring school prior to capture. Thus, the 3D spiral bubble net can control the herring schools more effectively. 


\section{References}

${ }^{1}$ J. Allen, M. Weinrich, W. Hoppitt, and L. Rendell, "Network-based diffusion analysis reveals cultural transmission of lobtail feeding in humpback whales," Science 340(6131), 485-488 (2013) doi: 10.1126/science.1231976.

${ }^{2}$ F. A. Sharpe and L. M. Dill, "The behavior of pacific herring schools in response to artificial humpback whale bubbles," Canadian Journal of Zoology 75(5), 725-730 (1997).

${ }^{3}$ D. Wiley, C. Ware, A. Bocconcelli, D. Cholewiak, A. Friedlaender, M. Thompson, and M. Weinrich, "Underwater components of humpback whale bubble-net feeding behaviour," Behaviour 148(5), 575-602 (2011).

${ }^{4}$ T. G. Leighton, S. D. Richards, and P. R. White, "Trapped within a wall of sound," Acoustics Bulletin 29, 24-29 (2004).

${ }^{5}$ C. G. D’Vincent, R. M. Nilson, and R. E. Hanna, "Vocalization and coordinated feeding behavior of the humpback whale in southeastern Alaska," Scientific Reports of the Whales Research Institute 36, 41-47 (1985).

${ }^{6}$ T. G. Leighton, D. Finfer, E. Grover, and P. R. White, "An acoustical hypothesis for the spiral bubble nets of humpback whales and the implications for whale feeding," Acoustics Bulletin 22(1), 17-21 (2007a).

${ }^{7}$ T. G. Leighton, D. C. Finfer, and P. R. White, "Cavitation and cetacean," Revista de Acústica 38(3-4), 37-81 (2007).

${ }^{8}$ T. G. Leighton, "From seas to surgeries, from babbling brooks to baby scans: The acoustics of gas bubbles in liquids," International Journal of Modern Physics B 18(25), 3267-3314 
(2004).

${ }^{9}$ R. S. Payne and S. McVay, "Songs of humpback whales," Science 173(3997), 585-597 (1971).

${ }^{10}$ R. A. Dunlop, M. J. Noad, D. H. Cato, and D. Stokes, "The social vocalization repertoire of east australian migrating humpback whales (megaptera novaeangliae)," The Journal of the Acoustical Society of America 122(5), 2893-2905 (2007).

${ }^{11}$ E. Jourdain and D. Vongraven, "Humpback whale (megaptera novaeangliae) and killer whale (orcinus orca) feeding aggregations for foraging on herring (clupea harengus) in northern norway," Mammalian Biology 86,27-32 (2017) doi: https://doi .org/10.1016/ j.mambio.2017.03.006.

${ }^{12}$ F. A. Sharpe, "Social foraging of the southeast alaskan humpback whale, megaptera novaengliae," Ph.D. dissertation, University of Washington, Seattle, Washington, 1984.

${ }^{13}$ T. G. Leighton and P. R. White, "Dolphin-inspired target detection for sonar and radar," Archives of Acoustics 39(3), 319-332 (2014).

${ }^{14}$ D. A. Mann, A. N. Popper, and B. Wilson, "Pacific herring hearing does not include ultrasound," Biology Letters 1(2), 158-161 (2005).

${ }^{15}$ A. N. Popper, D. T. T. Plachta, D. A. Mann, and D. Higgs, "Response of clupeid fish to ultrasound: a review," ICES Journal of Marine Science 61(7), 1057-1061 (2004) doi: 10.1016/j.icesjms.2004.06.005.

${ }^{16}$ J. M. Allen, J. Blaxter, and E. Denton, "The functional anatomy and development of the swimbladder-inner ear-lateral line system in herring and sprat," Journal of the Marine 
Biological Association of the United Kingdom 56(2), 471-486 (1976).

${ }^{17}$ T. A. Mooney, M. B. Kaplan, and M. O. Lammers, "Singing whales generate high levels of particle motion: implications for acoustic communication and hearing?," Biology letters 12(11), 20160381 (2016).

${ }^{18}$ T. G. Leighton, "What is ultrasound?," Progress in Biophysics and Molecular Biology 93(1-3), 3-83 (2007) <GotoISI>://WOS : 000243451700002 doi: 10.1016/j ·pbiomolbio. 2006.07 .026

${ }^{19}$ T. G. Leighton, S. Meers, and P. White, "Propagation through nonlinear time-dependent bubble clouds and the estimation of bubble populations from measured acoustic characteristics," in Proceedings of the Royal Society of London A: Mathematical, Physical and Engineering Sciences, The Royal Society (2004), Vol. 460, pp. 2521-2550.

${ }^{20}$ C. Wei, W. W. L. Au, D. R. Ketten, and Y. Zhang, "Finite element simulation of broadband biosonar signal propagation in the near- and far-field of an echolocating atlantic bottlenose dolphin (tursiops truncatus)," Journal of the Acoustical Society of America 143(5), 2611-2620 (2018) <GotoISI> : /WOS :000433050700024 doi: 10.1121/1.5034464.

${ }^{21}$ M. A. Ainslie and T. G. Leighton, "Review of scattering and extinction cross-sections, damping factors, and resonance frequencies of a spherical gas bubble," The Journal of the Acoustical Society of America 130(5), 3184-3208 (2011).

${ }^{22}$ K. W. Commander and A. Prosperetti, "Linear pressure waves in bubbly liquids: Comparison between theory and experiments," The Journal of the Acoustical Society of America 85(2), 732-746 (1989). 
${ }^{23}$ S. G. Kargl, "Effective medium approach to linear acoustics in bubbly liquids," The Journal of the Acoustical Society of America 111(1), 168-173 (2002).

${ }^{24} \mathrm{~A}$. Ingebrigtsen, "Whales caught in the north atlantic and other seas," Conseil Permanent International pour l'Exploration de la Mer. Rapports et Proces-Verbaux des Reunions 56, 123-135 (1929).

${ }^{25}$ C. M. Jurasz and V. P. Jurasz, "Feeding modes of the humpback whale, megaptera novaeangliae, in southeast alaska," Scientific Reports of the Whales Research Institute 31, 69-83 (1979).

${ }^{26}$ J. HW, R. Carter, D. Kraus, A. Mayo, and E. Winni, "Feeding behavior of the humpback whale, megaptera novaeangliae, in the western north atlantic," Fishery Bulletin $\mathbf{8 0}(2)$ (1982).

${ }^{27}$ M. T. Weinrich, M. R. Schilling, and C. R. Belt, "Evidence for acquisition of a novel feeding behaviour: lobtail feeding in humpback whales, megaptera novaeangliae," Animal behaviour 44(6), 1059-1072 (1992).

${ }^{28}$ W. W. L. Au, A. A. Pack, M. O. Lammers, L. M. Herman, M. H. Deakos, and K. Andrews, "Acoustic properties of humpback whale songs," Journal of the Acoustical Society of America 120(2), 1103-1110 (2006) doi: 10.1121/1.2211547.

${ }^{29}$ P. O. Thompson, W. C. Cummings, and S. J. Ha, "Sounds, source levels, and associated behavior of humpback whales, southeast alaska," The Journal of the Acoustical Society of America 80(3), 735-740 (1986). 
${ }^{30}$ J. S. Reidenberg and J. T. Laitman, "Blowing bubbles: An aquatic adaptation that risks protection of the respiratory tract in humpback whales (megaptera novaeangliae)," Anatomical Record-Advances in Integrative Anatomy and Evolutionary Biology 290(6), 569-580 (2007).

${ }^{31}$ T. G. Leighton, The acoustic bubble (Academic press, 2012), pp. 67-71.

${ }^{32}$ T. G. Leighton, J. Jiang, and K. Baik, "Demonstration comparing sound wave attenuation inside pipes containing bubbly water and water droplet fog," The Journal of the Acoustical Society of America 131(3), 2413-2421 (2012).

${ }^{33}$ T. G. Leighton, D. G. H. Coles, M. Srokosz, P. R. White, and D. K. Woolf, "Asymmetric transfer of CO2 across a broken sea surface," Scientific reports 8(1), 8301 (2018).

${ }^{34}$ T. G. Leighton, K. J. Fagan, and J. E. Field, "Acoustic and photographic studies of injected bubbles," European Journal of Physics 12(2), 77 (1991).

${ }^{35}$ T. G. Leighton and P. R. White, "Quantification of undersea gas leaks from carbon capture and storage facilities, from pipelines and from methane seeps, by their acoustic emissions,"

Proceedings of the Royal Society A: Mathematical, Physical and Engineering Sciences 468(2138), 485-510 (2011).

${ }^{36}$ J. Blackford, H. Stahl, J. M. Bull, B. J. Bergès, M. Cevatoglu, A. Lichtschlag, D. Connelly, R. H. James, J. Kita, D. Long, M. Naylor, K. Shitashima, D. Smith, P. Taylor, I. Wright, M. Akhurst, B. Chen, T. M. Gernon, C. Hauton, M. Hayashi, H. Kaieda, T. G. Leighton, T. Sato, M. D. J. Sayer, M. Suzumura, K. Tait, M. E. Vardy, P. R. White, S. Widdicombe, "Detection and impacts of leakage from sub-seafloor deep geological carbon dioxide stor- 
age," Nature climate change 4(11), 1011 (2014).

${ }^{37}$ B. J. Berges, T. G. Leighton, and P. R. White, "Passive acoustic quantification of gas fluxes during controlled gas release experiments," International Journal of Greenhouse Gas Control 38, 64-79 (2015).

${ }^{38}$ G. Qiao, X. Qing, W. Feng, S. Liu, D. Nie, and Y. Zhang, "Elastic feature of cylindrical shells extraction in time-frequency domain using biomimetic dolphin click," Journal of the Acoustical Society of America 142(6), 3787-3795 (2017) doi: 10.1121/1.5017835.

${ }^{39}$ S. Yoon, L. A. Crum, A. Prosperetti, and N. Lu, "An investigation of the collective oscillations of a bubble cloud," The Journal of the Acoustical Society of America 89(2), 700-706 (1991).

${ }^{40}$ M. Nicholas, R. Roy, L. Crum, H. Oguz, and A. Prosperetti, "Sound emissions by a laboratory bubble cloud," The Journal of the Acoustical Society of America 95(6), 3171$3182(1994)$.

${ }^{41}$ See Supplementary materials at [URL will be inserted by AIP] for [dynamically displaying the isosurface of sound pressure distribution.].

${ }^{42}$ See Supplementary materials at [URL will be inserted by AIP] for [dynamically displaying the slices of sound pressure distribution.].

${ }^{43}$ P. S. Enger, "Hearing in herring," Comparative Biochemistry and Physiology 22(2), 527538 (1967).

${ }^{44}$ G. Rieucau, L. D. Sivle, and N. Olav Handegard, "Herring perform stronger collective evasive reactions when previously exposed to killer whales calls," Behavioral Ecology 27(2), 
538-544 (2015).

${ }^{45}$ N. O. Handegard, A. D. Robertis, G. Rieucau, K. Boswell, and G. J. Macaulay, "The reaction of a captive herring school to playbacks of a noise-reduced and a conventional research vessel," Canadian journal of fisheries and aquatic sciences 72(4), 491-499 (2014).

${ }^{46}$ S. L. Nedelec, J. Campbell, A. N. Radford, S. D. Simpson, and N. D. Merchant, "Particle motion: the missing link in underwater acoustic ecology," Methods in Ecology and Evolution 7(7), 836-842 (2016).

${ }^{47}$ A. N. Popper and A. D. Hawkins, "The importance of particle motion to fishes and invertebrates," The Journal of the Acoustical Society of America 143(1), 470-488 (2018) doi: $10.1121 / 1.5021594$

${ }^{48}$ R. W. Nero, C. H. Thompson, and J. Michael Jech, "In situ acoustic estimates of the swimbladder volume of atlantic herring (clupea harengus)," ICES Journal of Marine Science 61(3), 323-337 (2004).

${ }^{49}$ D. E. Weston, "Sound propagation in the presence of bladder fish," Underwater acoustics 2, 55-88 (1967).

${ }^{50}$ J. H. W. Hain, G. R. Carter, S. D. Kraus, C. A. Mayo, and H. E. Winni, "Feeding behavior of the humpback whale, megaptera noveangliae, in the western north atlantic," Fishery Bulletin 80(2), 259-268 (1982).

${ }^{51}$ See Supplementary materials at [URL will be inserted by AIP] for [dynamically displaying the slices of sound pressure distribution.]. 
JASA

$777{ }^{52}$ See Supplementary materials at [URL will be inserted by AIP] for [dynamically displaying 778 the slices of particle velocity distribution.]. 